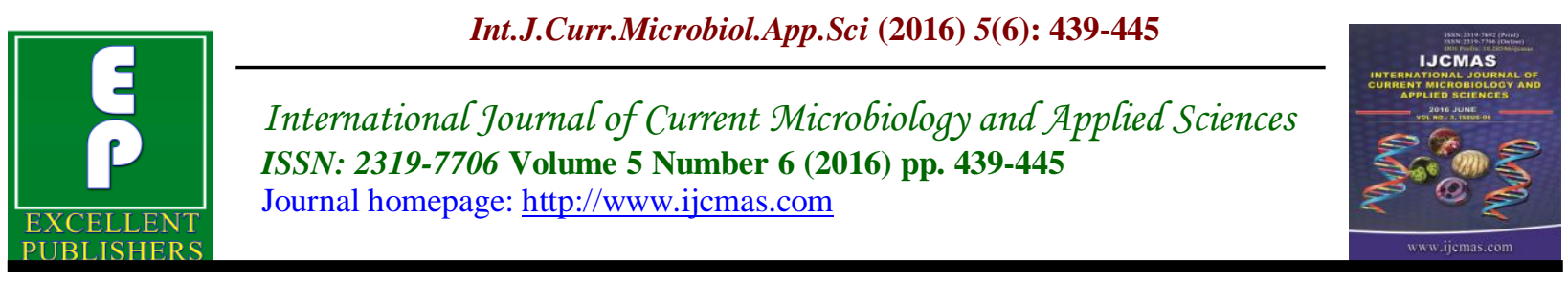

Original Research Article

http://dx.doi.org/10.20546/ijcmas.2016.506.051

\title{
Sero-Prevalence of Dengue in Tertiary Care Hospital in Delhi
}

\author{
H. Lall, P. Gupta, M. Debbarma, P. Sharma, S.K. Ansari, M. Jais* and R. Kaur \\ Department of Microbiology, Lady Hardinge Medical College, New Delhi, India
}

*Corresponding author

\begin{abstract}
A B S T R A C T
Keywords

Sero-Prevalence,

Dengue,

IgM antibody

and

NS1 antigen.

Article Info

Accepted:

20 May 2016

Available Online:

10 June 2016

This retrospective study was done in year 2015 to find out the seroprevalence of dengue IgM antibody and NS1 antigen positivity, in a tertiary care hospital in New Delhi, India. Blood samples from clinically suspected dengue cases admitted in the Medicine and Paediatrics, Obsteritics and Gynaecology departments of a tertiary care hospital were collected. Samples from fever cases $<5$ days duration were tested for dengue NS1 antigen employing Pan Bio (Australia) NS1 ELISA kit. Serum samples of $\geq 5$ days fever were tested for dengue-specific IgM by MAC ELISA test kit prepared by the National Institute of Virology Pune. Results of the 3163 samples tested, $646(20.4 \%)$ were positive for dengue IgM. Majority (47.86 $\%$ ) of cases were detected in the month of Aug-Nov. The most common affected age group was 11 to $30 \mathrm{yr}$ with female preponderance. 265 were tested positive by IgM Elisa (M-126, 47.5\%, F-139, 52.4\%) and 381 by NS1 Ag Elisa (M-163, $42.7 \%$, F-218, (57.2\%). Total number of male patients was 1266 and female patients were 1897. Rapid increase in the dengue cases in 2015 became a public health concern as majority of cases were affecting the young adolescents and adults. Most of the cases were reported in monsoon and post-monsoon periods indicating increased vector transmission
\end{abstract}

\section{Introduction}

Dengue viruses (DV) belong to the family Flaviviridae, and there are four serotypes of the virus referred to as DV-1, DV-2, DV-3, and DV-4. It is transmitted mainly by Aedes aegypti mosquito and also by A. albopictus. DV is a positive-stranded encapsulated RNA virus and is composed of three structural protein genes, which encode the nucleocapsid or core (C) protein, a membrane-associated (M) protein, an enveloped (E) glycoprotein, and seven nonstructural (NS) proteins.
(Bandyopadhyay et al., 2013). Dengue is a complex disease with a wide spectrum of clinical presentation following the period of incubation, most patients experience a sudden onset of fever which can remain for 2-7 days and is often accompanied with symptoms such as myalgia, arthralgia, anorexia, sore throat, headaches, and a macular skin rash. It is during this period that differentiating dengue from other febrile diseases proves troublesome. The majority of people experience a self-limiting clinical 
course, which does not progress to the severe forms of dengue, dengue hemorrhagic fever (DHF), or dengue shock syndrome (DSS). Secondary dengue infections or particularly virulent viral strains are two factors thought to be associated with increased risk of severity (Gubler, 2011). In severe cases, thrombocytopenia and increased vascular permeability can result in hemorrhagic and shock complications. Currently, neither a vaccine nor specific antiviral therapy exists (Wilder-Smith et al., 2011). However, with prompt case detection and appropriate clinical management, including initiation of intravenous rehydration, the case fatality of severe dengue can be lower than $1 \%$.At present, due to a lack of available treatment, control of dengue focuses on effective vector control methods, which are limited (Gibbons et al., 2002) DV causes subclinical infection to a mild self-limiting disease, the dengue fever (DF), a severe disease that maybe fatal, and the dengue haemorrhagic fever/dengue shock syndrome (DHF/DSS).

In 2012, dengue was classified by the World Health Organization (WHO) as the 'most important mosquito-borne viral disease in the world. Due to significant geographic spread of the virus and its vector into previously unaffected areas and the subsequent costly burden of disease it brings IgM antibody is the first immunoglobulin isotype to appear. In a suspected case of dengue, the presence of antidengue $\operatorname{IgM}$ antibody suggests recent infection. Antidengue IgM detection using enzyme-linked immunosorbent assay (ELISA) represents one of the most important advances and has become an invaluable tool for routine dengue diagnosis. Specifically, MAC ELISA (IgM antibody capture ELISA) diagnosis is based on detecting denguespecific IgM (Vaughn et al., 2000)

\section{Materials and Methods}

The Blood samples were collected from clinically suspected acute dengue fever cases(fever $<4$ days). Suspected cases of dengue were defined as individuals who presented with symptoms of dengue fever and warning sign. The latter symptoms included the following : decrease in platelet count, vomiting, mucosal bleeding, lethargy, weak pulse rate, hypotension. The study was conducted between January-December2015 in Department of Microbiology at Tertiary care Hospital in Delhi. The samples were screened for the presence of dengue-specific IgM antibody capture enzyme linked immunsorbent assay(MAC-ELISA), using a kit prepared by the National Institute of virology, Pune, India, (as an integral part of the National Vector Borne Disease Control Programme), strictly following the manufacurer's protocol (Cecilia, 2011). NS1 $\mathrm{Ag}$ was detected in the sera by PanBio NS1Ag ELISA kit. Haematological parameters (leucocyte count, $\mathrm{Hb}$ and platelet count) of dengue positive patients were also observed and correlated.

\section{Results and Discussion}

Total of 3163 sera samples were tested and dengue positive were 646(20.4\%) samples. 265 were tested positive by IgM Elisa (M$126,47.5 \% \mathrm{~F}-139,52.4 \%$ )and 381 by NS1 Ag Elisa (M-163, 42.7\%, F-218, (57.2\%). Total number of male patients were 1266 and female patients were 1897.

Fig2 and 3 shows distribution of the number of the dengue cases in various age groups in either sex for NS1Ag and IgM tests respectively. It clearly shows that the highest number of cases belonged to the age group 11-30 year and females clearly outnumbered males. 
As the outbreak of dengue mainly occurred in the months of August to November in 2015.Fig 4shows the weekly distribution of cases was reported from the $1^{\text {st }}$ week of September to almost mid-October for both seromarkers of dengue (IgM and NS1 antigen).

Fig1 month wise distribution of the total dengue cases in year 2015.The highest number of cases were recorded during the monsoon and post monsoon periods. The number of affected cases declined with the onset of winter. Fig 5 shows location wise distribution of dengue cases in Delhi and NCR. Dengue is one of the re-emerging viral inections. India witnesses dengue fever outbreak every year. There has been a considerable increase in the geographic spread, number of cases and severity of the disease in the past four decades. Reason may be there is drastic change in the environment due to rapid urbanization and increase in transportation facilities in several parts of India. Delhi has had several outbreaks of dengue caused by various dengue virus types in 1967,1970,1982,1988 and 1996 (Dar, 2006).

Fig.1 Monthly distribution of dengue positive cases

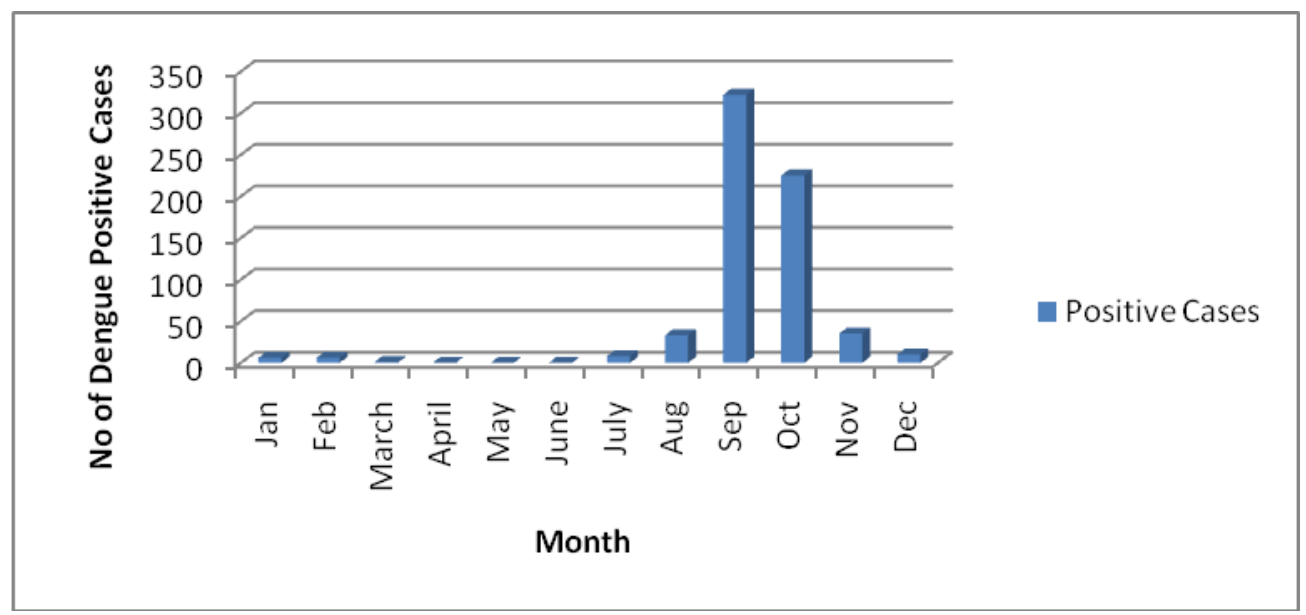

Fig.2 Showing NS1 positivity at different age Group and Gender .

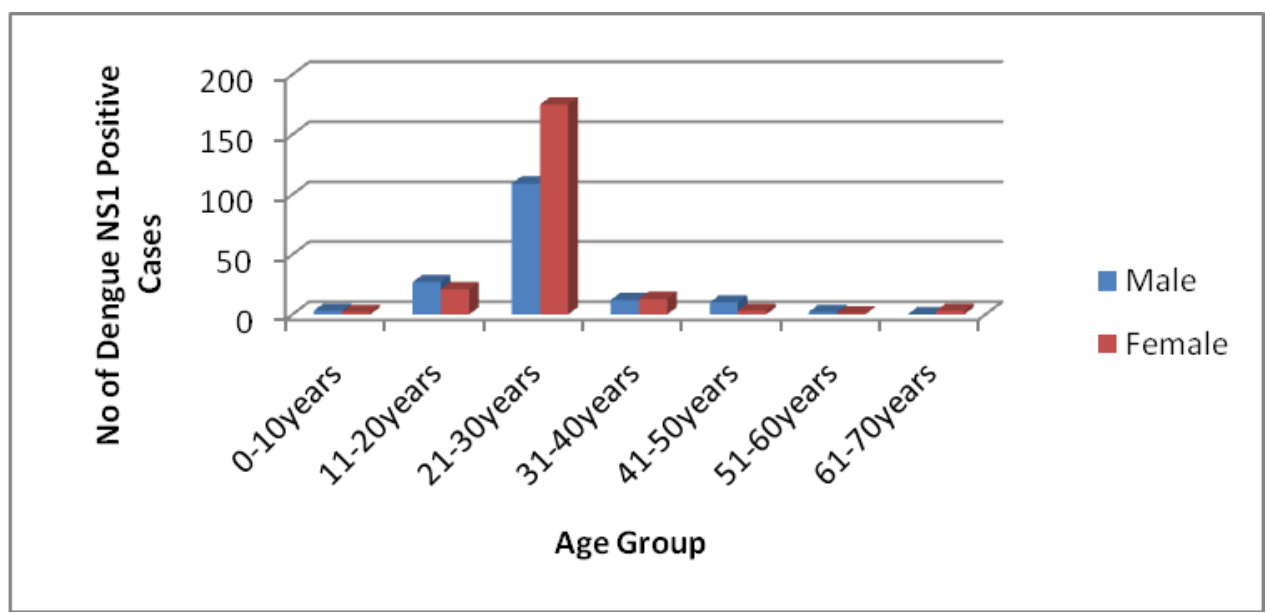


Fig.3 Showing IgM positivity at different Age Group and Gender

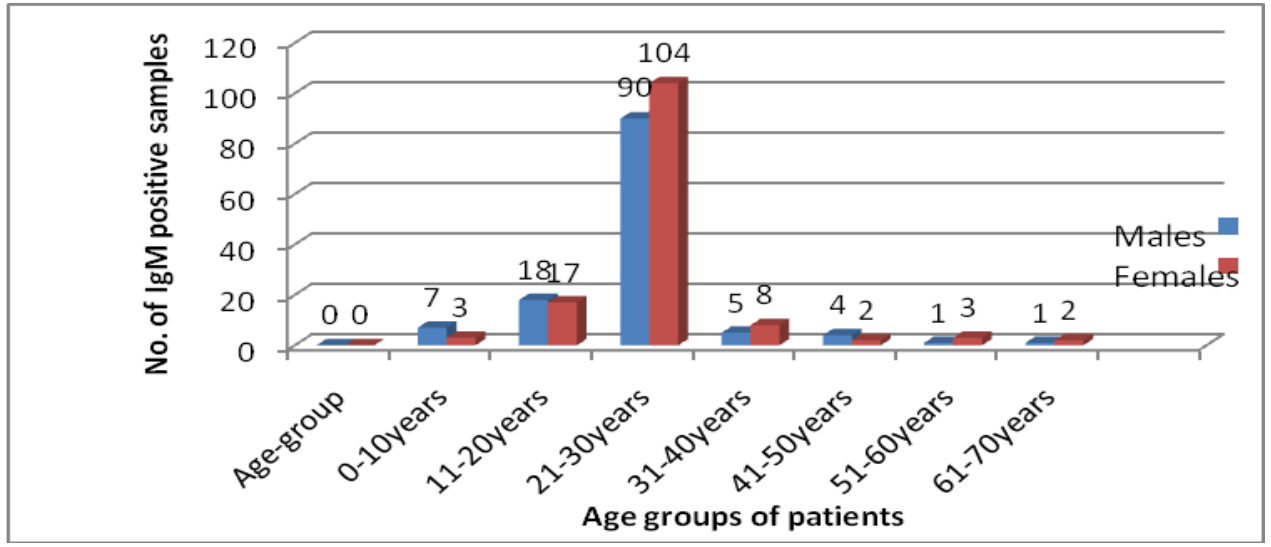

Fig.4 Shows Week wise distribution of dengue positive cases.

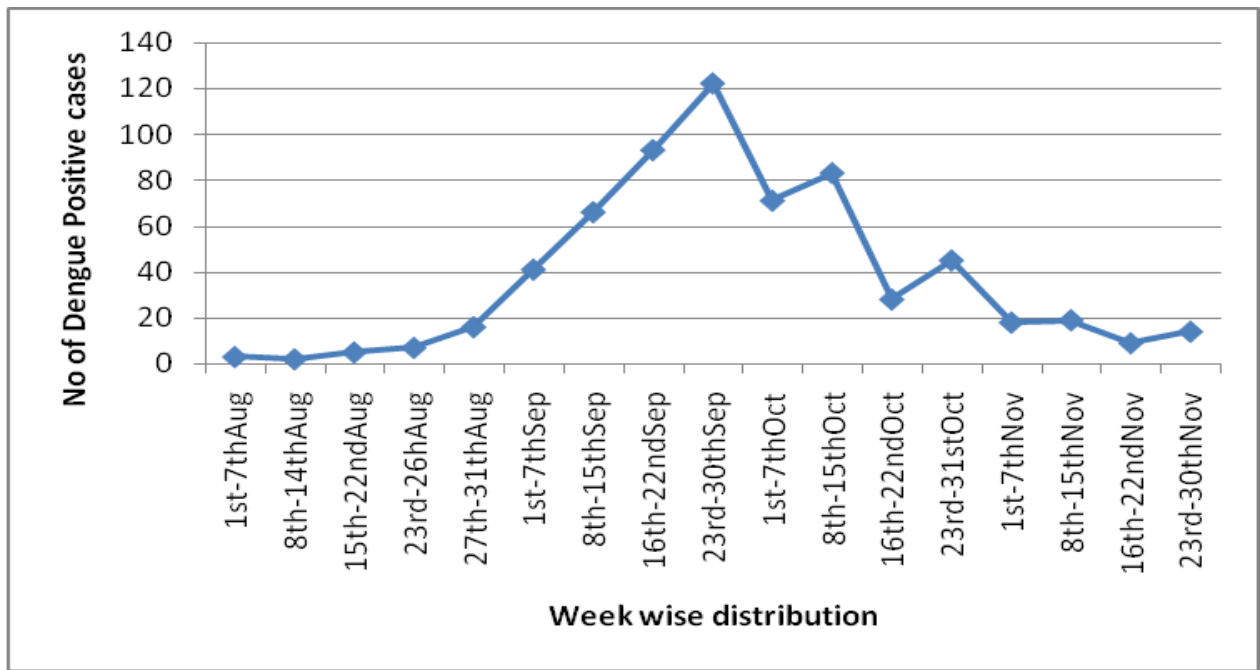

Fig.5 Area wise distribution of Dengue Positive cases.

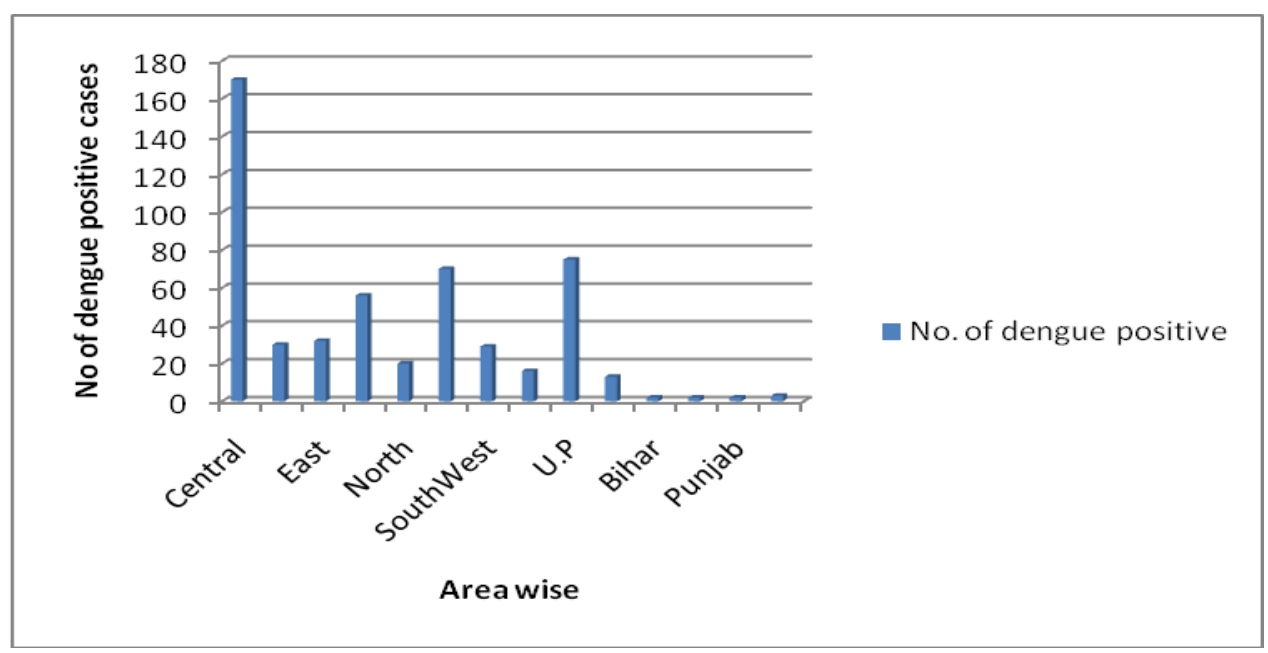


In present study, 20.4\% (646) of the suspected cases were found serologically positive for dengue. The remaining $79.6 \%$ cases were serologically negative for dengue. Those patients might be suffering from other illnesses like Malaria, Chikungunya, Measles, rubella. In our study, the highest number of cases belonged to the age group 11-30 year and females clearly outnumbered males which was in concordance with Gupta et al., and Kumaria et al study which also showed maximum cases in age group of 21-30 years but with male prepordance. The young adults getting more affected reflects the presence of nonimmune adult population falling prey to the circulating serotype of dengue virus. Another reason could be these young adults involved in more of outdoor activities, more exposed for mosquito bites.

Sarkar et al., however, reported maximum cases in the age group 0-10 years with female preponderance. Concordance study, was also observed by Chakravarti et al., who found highest seropositivity for dengue in female gender for both NS1antigen and IgM Elisa. Higher prevalence among females might be due to their more outdoor activities when compared to males, resulting in more exposure to day-time biting mosquitoes (Madhulika et al., 2013). The vector mosquitoes (Aedes sp.) are mainly domestic and peridomestic in nature and females/ house wives have a greater chance of exposure to mosquito bites. The lower case positivity rates in males may be attributed to lower reporting rate. However, contrasting results were reported by Agarwal et al., and wali et al, found twice number of male patients as compared to female patients.

In present study, the majority of the cases were reported during the monsoon and post monsoon seasons, which indicated an active viral transmission during monsoon and postmonsoon season. Aedes aegypti vector mosquito which breeds during the rainy season and continues to reproduce and add to its number usually till the winter season.

The maximum dengue positive cases were observed from the $1^{\text {st }}$ week of September to almost mid-October for both seromarkers of dengue.

According to areawise, the majority of dengue positive cases were reported from Central Delhi (paharganj, Nabikarim, rajendernagar etc) followed by West Delhi and South West Delhi. Few dengue positive cases were also reported from Gaziabad, Faridabad, Punjab and Gurgoan in our study. Since there is increase in urbanization, increase travel among people to Central Delhi in need of better job facilities and trades, might be responsible for spread of the disease.

Since there is no specific drug and vaccine available and treatment is only supportive. Therefore control measures to stop vector and disease is extremely important. As most of the cases reported during the postmonsoon period, continue and coordinated efforts should be made to control the transmission of vector and prevent the outbreak. A long term serosurveillance study may help to provide more information about the intensity, seasonal incidence and seasonal effect.

In conclusion, rapid increase in the dengue cases in 2015 became a public health concern as majority of cases were affecting the young adolescents and adults. Most of the cases were reported in monsoon and post-monsoon periods indicating increased vector transmission.

\section{References}

Agarwal, R., Kapoor, S., Nagar, R., Misra, A., Tandon, R., Mathur, A., et al. 
1999. A clinical study of the patients with dengue hemorrhagic fever during the epidemic of 1996 at Lucknow, India. Southeast Asian J. Trop. Med. Public Health, 30: 735-40.

Bandyopadhyay, B., Bhattacharyya, I., Adhikary, S., Konar, J., Dawar, N., Chakravarty, A.A. 2013. Comprehensive Study on the 2012 Dengue Fever Outbreak in Kolkata, India .Hindawi Publishing Corporation ISRN Virology Volume 2013, Article ID 207580, 5 pages

Cecilia, D., M. B. Kakade, A. B. Bhagat et al. 2011. "Detection of dengue-4 virus in Pune, Western India after an absence of 30 years-its association with two severe cases," Virol. J., vol. 8, article 46.

Chakravarti, A., P. Roy, S. Malik, et al. 2016. A study of gender related differences in laboratory Characteristics of dengue fever. IJMM, 34(1): 82-84.

Chakravarti, A., R. Kumaria. 2005. "Ecoepidemiological analysis of dengue infection during an Outbreak of dengue fever, India," Virol. J., vol. 2, article 32.

Chakravati, A., M. Matlani, B. Kashyap, A. Kumar. 2012. Awareness of changing trends in Epidemiology of dengue fever is essential for epidemiological surveillance. IJMM, 30(2): 232-6.

Dar, L., Gupta, E., Narang, P., Broor, S. 2006. Cocirculation of Dengue Serotypes, Delhi, India. Emerg. Infect. Dis., 12: 352-3.

Evelyn, N., Murray, A., Mikkel, B.Q., Annelies, S. 2013. Epidemiology of dengue: past, Present and future prospects Clinical Epidemiol., 5: 299-309.

Gibbons, R.V., Vaughn, D.W. 2002.
Dengue: an escalating problem. BMJ, 324(7353): 1563-1566.

Gubler, D.J. 2011. Dengue, Urbanization and Globalization: The Unholy Trinity of the 21(st) Century. Trop. Med. Health., 39(Suppl 4): 3-11.

Gupta, E., L. Dar, G. Kapoor, S. Broor. 2006. The changing epidemiology of dengue in Delhi, India. Virol. J., vol.3, article 92.

Hati, A.K. 2006. Studies on dengue and dengue haemorrhagic fever (DHF) in West Bengal State, India, $J$. Communicable Dis., vol. 38 , no. 2 , pp. 124-129.

http://dx.doi.org/10.5402/2013/207580.

Madhulika, Mistry, Yogesh, Goswami, Rajesh, K., Chudasama, Dhara, Thakkar. 2015. Epidemiological and demographic characteristics of dengue disease at a tertiary care centre In Saurashtra region during the year 2013. J. Vector Borne Dis., 52: 299-303.

Minh, A.N., Joacim Rocklo". v. 2014. Epidemiology of dengue fever in Hanoi from 2002 to 2010. And its meteorological determinants Global Health Action 2014. \#2014

Ramana, K.V. 2014. Dengue viral infection: Focus on epidemiology, laboratory diagnosis, and Management and control measures. J. Appl. Environ. Microbiol., 2: 249-52.

Reiter, P. 2001. Climate change and mosquito-borne disease," Environ. Health Perspectives, vol. 109, supplement 1, pp. 141-161.

Sarkar, A., D. Taraphdar, S. Chatterjee. 2012. "Molecular typing of dengue virus circulating in Kolkata, India in 2010," J. Trop. Med., vol. 2012, Article ID 960329, 5pages.

Siraj, A., Khan, Prafulla Dutta, Rashmee Topno, Monika Soni, Mahanta, J. 2014. Dengue Outbreak in a Hilly 
State of Arunachal Pradesh in Northeast India. Scientific World J., Volume 2014, Article ID 584093.

Vaughn, D.W., S. Green, S. Kalayanarooj $e t$ al., 2000. Dengue viremiaiter, antibody response Pattern, and virus serotype correlate with disease severity," J. Infect. Dis., vol. 181: no.1, pp. 2-9.

Wali, J.P., Biswas, A., Handa, R., Aggarwal, P., Wig, N., Dwivedi, S.N. 1999. Dengue haemorrhagic fever in adults: A prospective study of 110 cases. Trop. Doct., 29: 27-30.

Wilder-Smith, A., Foo, W., Earnest, A., Sremulanathan, S., Paton, NI. 2004. Seroepidemiology of dengue. In the adult population of Singapore. Trop.
Med. Int. Health, 9(2): 305-308.

Wilder-Smith, A., Ooi, E.E., Vasudevan, S.G., Gubler, D.J. 2010. Update on dengue: epidemiology, virus Evolution, antiviral drugs, and vaccine development. Curr. Infect. Dis. Rep., 12(3): 157-164.

World Health Organization (WHO). 2012. Global Strategy for Dengue Prevention and Control, 2012-2020. Geneva: WHO Press.

World Health Organization. 2001. Iron Deficiency Anaemia: Assessment, Prevention and Control, A Guide for Programme Managers. Geneva: World Health Organization.

\section{How to cite this article:}

Lall, H., M. Jais and Gupta, P. 2016. Sero-Prevalence of Dengue in Tertiary Care Hospital in Delhi. Int.J.Curr.Microbiol.App.Sci. 5(6): 439-445. doi: http://dx.doi.org/10.20546/ijcmas.2016.506.051 